\title{
Omental torsion, a rare cause of acute abdomen
}

\author{
Shivani Joshi, ${ }^{1}$ Gary Andrew Cuthbert, ${ }^{2}$ Rajab Kerwat ${ }^{2}$
}

\begin{abstract}
Kent and Canterbury Hospital Canterbury, UK

${ }^{2}$ Department of General

Surgery, Lewisham and

Greenwich NHS Trust, London, UK
\end{abstract}

\section{Correspondence to}

Dr Shivani Joshi,

shivani.joshi@doctors.org.uk

Accepted 4 December 2015

\section{SUMMARY}

Omental torsion is a rare cause of acute abdominal pain. We report a case of omental torsion in a 7-year-old girl, who presented with right iliac fossa pain. The patient underwent an open appendicectomy, during which a normal appendix was identified and a diagnosis of omental torsion was made. The affected segment of omentum was resected along with the appendix. The patient made an uncomplicated recovery and was discharged on day 1 postoperatively. A normal appendix in the presence of omental torsion is found in $0.1 \%$ of all appendicectomies in children. We suggest routine visualisation of the omentum in the presence of a normal appendix during appendicectomy.

\section{BACKGROUND}

Eitel first described primary omental torsion in 1899. ${ }^{1}$ There have not yet been any definitive guidelines to aid diagnosis. Reported cases describe characteristic appearances on radiological imaging; however, surgical exploration remains the definitive gold standard for intraoperative diagnosis and therapeutic management. Increased awareness of omental torsion as a differential diagnosis for acute abdominal pain will encourage routine inspection of the omentum intraoperatively. It may act to advocate the use of laparoscopy in suitable patients, in order to take advantage of its diagnostic value in exploring the abdominal cavity in the presence of a normal appendix.

\section{CASE PRESENTATION}

A 7-year-old girl was admitted to the local accident and emergency department, reporting acute onset of localised non-migratory right-sided abdominal pain and fever, for which she had been sent home from school the same day. The pain was not eased by paracetamol, and accompanying symptoms included fevers, rigours and dysuria. There was no significant anorexia, nausea or vomiting. The patient did not report any upper respiratory tract symptoms or change in bowel habit. Her medical history included a full term normal vaginal birth, with no history of childhood illness or surgery. She was of normal body mass index and had up-to-date vaccinations.

The patient was referred by the paediatricians to the surgical team. On examination of the vital signs, she was normotensive, but feverish $\left(38.0^{\circ} \mathrm{C}\right)$ and tachycardic $(151 \mathrm{bpm})$. On examination of the abdomen, she was noted to be tender over the right iliac fossa, with localised rebound tenderness and guarding. Examinations of the remaining systems were unremarkable. Additional examination of both hips confirmed no bony tenderness, and no pain on active or passive movements.

\section{INVESTIGATIONS}

Routine bloods on admission showed a moderate leucocytosis; a white cell count of $18.1 \times 10^{9} / \mathrm{L}$, neutrophils of $13.4 \times 10^{9} / \mathrm{L}$ and a $\mathrm{C}$ reactive protein of $51 \mathrm{mg} / \mathrm{L}$. A urine dipstick was found to be negative for nitrates, blood, protein and white cells, ruling out urinary tract infection as a possible cause for her symptoms.

A clinical diagnosis of acute appendicitis was made by the surgical team, with a plan to proceed with open appendicectomy. Imaging was not deemed necessary given the strong clinical evidence supporting a diagnosis of appendicitis. However, if the presentation had been less tangible, an ultrasound could have been offered to aid preoperative diagnosis.

\section{DIFFERENTIAL DIAGNOSIS}

The differential diagnoses considered in this case by the surgical team included acute appendicitis, Meckel's diverticulum, mesenteric adenitis and ovarian pathology.

Right iliac fossa pain in a premenarche female, with associated leucocytosis, fever and vomiting, is often a classical presentation of acute appendicitis, and is treated by an appendicectomy; this remains the most common cause for emergency surgery today, via either laparoscopy or open technique.

Intraoperatively, when no inflammation is found surrounding the appendix, it is routine to check for other pathology, such as Meckel's diverticulum. Meckel's diverticulum is a congenital bulge found in the terminal ileum two feet proximal to the ileocaecal valve, representing a vestigial remnant of the omphalomesenteric duct. ${ }^{2}$ It is present in approximately $2 \%$ of the population and is mostly asymptomatic; however, if symptomatic, it commonly occurs before the age of 2 years, presenting typically with painless rectal bleeding and right iliac fossa pain mimicking that of acute appendicitis. ${ }^{2}$ Definitive treatment of a symptomatic Meckel's diverticulum would involve surgical excision of the diverticulum with or without the adjacent ileal segment. $^{2}$

In this patient, the presence of a normal appendix and terminal ileum on inspection ruled out these differentials as a cause for her abdominal pain. Other possible paediatric causes of right iliac fossa pain in the presence of a normal appendix include mesenteric adenitis, urinary tract infection and ovarian pathology. Mesenteric adenitis involves inflammation of the mesenteric lymph nodes, typically causing pain in the right iliac fossa. ${ }^{3}$ It is 
differentiated by preceding symptoms of upper respiratory tract infection.

The most common ovarian pathologies seen in paediatric females are ovarian cysts or less commonly, malignant neoplasms. The diagnosis would normally be made by preliminary imaging or at diagnostic laparoscopy. ${ }^{4}$

Omental torsion is a rare differential for acute abdominal pain, clinically mimicking acute appendicitis-presenting typically with constant non-radiating pain in the right iliac fossa, and occasionally with associated low-grade fevers, moderate leucocytosis and vomiting. Fifty per cent of patients are found to have omental torsion present with an abdominal mass and localised peritonitis. ${ }^{5}$ They are found to be less systemically unwell than those with acute appendicitis, ${ }^{6}$ although most diagnoses are made intraoperatively in the presence of a normal appendix.

\section{TREATMENT}

This particular case took place in a district general hospital lacking specialist paediatric services. On the basis of clinical signs and laboratory investigations, there was a high clinical suspicion of acute appendicitis. A decision was made to proceed with appendicectomy. The first steps in management were intravenous fluids and antibiotics. The patient was kept nil by mouth and preparations were made for theatre. The team proceeded with an open appendicectomy via a Lanz incision. On exploration, the appendix was normal in the presence of a patch of torted ischaemic omentum (figure 1). This was found arising from the right side of the transverse colon, with consequent ischaemia of the omentum. The left side of the omentum was found to be normal, with only a small amount of haemoserous fluid. The omentum was clipped and divided proximal to the point of torsion. The fluid was collected and sent for microscopy, and the omentum sent for histology. The appendix was also removed in the usual way.

The definitive treatment for omental torsion is resection of the involved segment. The literature regarding methodology remains conflicting. Laparoscopy is considered by some to be the most appropriate technique for both diagnosis and treatment in cases where the diagnosis is doubtful. ${ }^{7}$ However, it has also been argued that although exploratory laparoscopy may aid diagnosis, laparotomy should be the definitive method for omental excision. ${ }^{8}$ The importance of establishing a preoperative diagnosis is emphasised in order to allow conservative management where possible.

The use of laparoscopy confers various benefits including a reduction in postoperative pain, shorter hospital stays and a

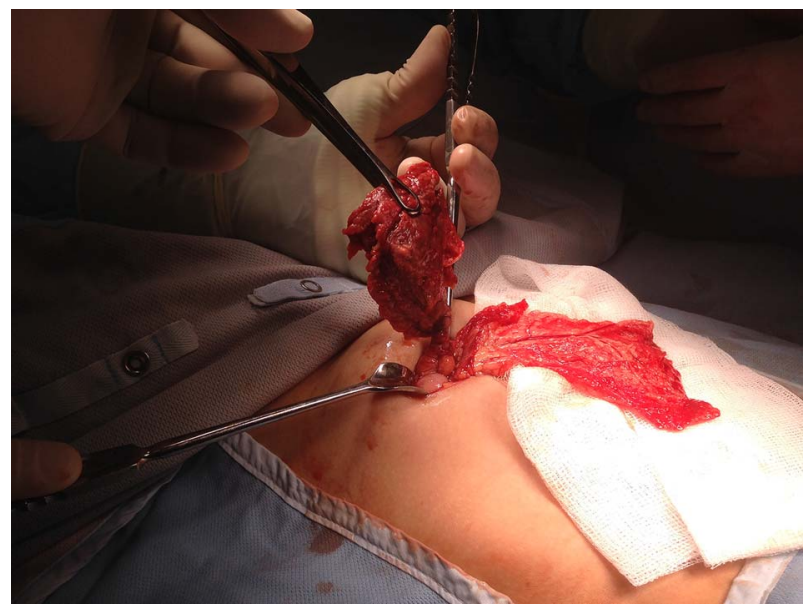

Figure 1 The congested, torted omentum on a narrow pedicle, delivered through the incision site prior to resection. reduced incidence of wound complications and scarring. The disadvantages include loss of touch sensation, difficulty in controlling bleeding and a two-dimensional image. ${ }^{9}$ In this circumstance, patient safety being of utmost importance, the skill and preference of the operator was considered and after discussion with the family, the decision was made to proceed with an open procedure.

\section{OUTCOME AND FOLLOW-UP}

The histology from the omental section showed focal necrosis of the omentum with acute inflammation and haemorrhage consistent with torsion. The sections from the appendix were consistent with periappendicitis plus inflammation of the serosa. There was no evidence of acute suppurative appendicitis.

The patient remained haemodynamically stable and afebrile throughout her recovery and was discharged the following day. Further follow-up was not required.

\section{DISCUSSION}

The greater omentum, embryonically, is derived from the dorsal mesentery. It is a sheet consisting of four layers of peritoneum, suspended from the greater curvature to cover the surrounding organs. ${ }^{1}$

Omental torsion occurs as the omentum twists around a pivotal point on its long axis. The most common point of torsion is around the distal right epiploic artery due to the increased length and mobility on the right side of the omentum. ${ }^{1}$ This results in venous obstruction, causing further oedema and vascular compromise, consequently leading to necrosis and adhesion formation. ${ }^{10}$

Omental torsion can be primary or secondary. Primary torsion forms one-third of cases, with no underlying causative pathology or distal fixation. ${ }^{6}$ Precipitating factors include trauma, obesity and sudden changes in body position or excessive exertion, that lead to an increase in abdominal pressure. ${ }^{10}$ Secondary causes can include adhesions from previous surgeries, hernias and other foci of inflammation. ${ }^{11}$ These cause a distal fixation, resulting in bipolar torsion. ${ }^{12}$ In extremely rare cases, the omentum has been noted to infarct without any evidence of torsion, a phenomenon known as primary idiopathic segmental infarction. $^{13}$

Since the first diagnosis of omental torsion by Eitel in 1899, primary omental torsion has been found in 1\% of appendicectomies carried out for suspected appendicitis in children. ${ }^{14}$ Patients typically present with non-radiating pain in the right iliac fossa and a moderate leucocytosis. There is often associated low-grade fever, anorexia, nausea or vomiting. Fifty per cent of patients with large segment involvement are found to have a palpable mass on abdominal examination, with localised peritonitis. ${ }^{5}$ Retrospective studies have shown that patients found to have omental torsion have been less systemically unwell compared to those with acute appendicitis. ${ }^{6}$ Patients typically present within the third to fifth decades, with a slightly higher prevalence in males $(3: 2){ }^{6}$

In cases where the clinical picture is equivocal, ultrasound and CT have been shown to have a role in diagnosis. ${ }^{10}$ CT scans in positive cases typically show hyperattenuated streaks of fat beneath the parietal peritoneum in the affected segment, with associated thickening of the overlying anterior abdominal wall. ${ }^{5}$ There is often a characteristic whirling pattern of the mesentery, with an accumulation of serosanguinous fluid within the peritoneum. ${ }^{5}$ Ultrasound imaging is useful in cases presenting with a palpable mass. A hyperechoic, non-compressible mass is typically shown with localised point tenderness. ${ }^{10}$ 
Diagnostic laparoscopy in suitable patients remains the investigation of choice for patients with atypical presentations, allowing intraoperative diagnosis and treatment. ${ }^{15}$ However, it has been argued that laparotomy should be the definitive method for omental excision. ${ }^{2}$

Findings include an infarcted segment of omentum and, often, a large volume of sterile, serosanguinous fluid within the peritoneal cavity, due to haemorrhagic extravasation. ${ }^{15}$ The normal appendix is usually removed to prevent future difficulties in diagnosis. ${ }^{6}$

Our recommendation for appropriate surgical management is routine inspection of the omentum in the presence of a normal appendix and terminal ileum. Visualisation of the omentum via a Lanz incision can be challenging in overweight children and adolescents. In these patients, a move towards laparoscopy will improve the chances of making accurate diagnoses.

\section{Learning points}

- Increased awareness of omental torsion as a differential diagnosis for acute abdominal pain is needed.

- Routine inspection of the omentum in the presence of a normal appendix during appendicectomy is required.

- It is important to acquire preoperative imaging when appropriate.

- The diagnostic value of laparoscopy in patients with equivocal clinical signs is highlighted.

Acknowledgements The authors would like to thank the General Surgery Department, Queen Elizabeth Hospital, Lewisham and Greenwich NHS Trust.
Contributors RK was the primary surgeon for the case, assisted by GAC, a core surgical trainee. SJ observed the case. The report was written by SJ, with further editing by GAC, prior to final approval by RK.

Competing interests None declared.

\section{Patient consent Obtained.}

Provenance and peer review Not commissioned; externally peer reviewed.

\section{REFERENCES}

1 Tsironos A, Nikolaos Z, Bali C, et al. Acute abdomen due to primary omental torsion: case report. J Emerg Med 2013;44:45-8.

2 Sagar J, Kumar V, Shah DK. Meckel's diverticulum: a systematic review. J R Soc Med 2006;99:501-5.

3 Yang WC, Chen CY, Wu HP. Etiology of non-traumatic acute abdomen in pediatric emergency departments. World J Clin Cases 2013;1:276-84.

4 De Silva KS, Kanumakala S, Grover SR, et al. Ovarian lesions in children and adolescents - an 11 year review. J Pediatr Endocrinol Metab 2004;17:951-7.

5 Efthimiou M, Kouritas VK, Fafoulakis F, et al. Primary omental torsion: report of two cases. Surg Today 2009;39:64-7.

6 Breunung N, Strauss P. A diagnostic challenge: primary omental torsion and literature review - a case report. World J Emerg Surg 2009;4:40.

7 Alexiou K, Ioannidis A, Drikos I, et al. Torsion of the greater omentum: two case reports. J Med Case Rep 2015;9:160.

8 Ghosh Y, Arora R. Omental torsion. J Clin Diagn Res 2014;8:1-2.

9 Tam PK, Laparoscopic surgery in children. Arch Dis Child 2000;82:240-3.

10 Theriot J, Sayat J, Franco $S$, et al. Childhood obesity: a risk factor for omental torsion. Paediatrics 2003;112:460.

11 Sarvepalli R, Kyle SM, Omental torsion: a rare cause of acute abdomen. $N$ Z Med J 2010;123:81-3.

12 Jeganathan $\mathrm{R}$, Epanomeritakis $\mathrm{E}$, Diamond T. Primary torsion of the omentum. Ulster Med J 2002;71:76-7.

13 Al-Jaberi T, Gharaibeh K, Yaghan R. Torsion of abdominal appendages presenting with acute abdominal pain. Ann Saudi Med 2000:20:211-13.

14 Chan KW, Chow CS, Tam YH, et al. Laparoscopy: an excellent tool in the management of primary omental torsion in children. J Laparoendosc Adv Surg Tech A 2007:17:821-4.

15 Khalid K, Somuah T, Aziz E, et al. Primary omental torsion in children: case report. Pan Afr Med J 2013;14:57.

Copyright 2016 BMJ Publishing Group. All rights reserved. For permission to reuse any of this content visit

http://group.bmj.com/group/rights-licensing/permissions.

BMJ Case Report Fellows may re-use this article for personal use and teaching without any further permission.

Become a Fellow of BMJ Case Reports today and you can:

- Submit as many cases as you like

- Enjoy fast sympathetic peer review and rapid publication of accepted articles

- Access all the published articles

- Re-use any of the published material for personal use and teaching without further permission

For information on Institutional Fellowships contact consortiasales@bmjgroup.com

Visit casereports.bmj.com for more articles like this and to become a Fellow 